\title{
Jogo de quadros na perspectiva de Régine Douady
}

\author{
Game of presentment in perspective Regine Douady
}

Paulo Jorge Magalhães Teixeira pjuff@yahoo.com.br

\begin{abstract}
Resumo
Este trabalho objetiva refletir acerca de questões relativas ao ensino e à aprendizagem de conceitos matemáticos. Muitas vezes - particularmente em geometria - quando um problema é proposto há necessidade de se mudar de contexto no qual ele é apresentado de modo a poder resolvê-lo. A essa passagem de um contexto para outro Régine Douady chama de mudança de quadro (Jogo de quadros). Por vezes, fazer uma mudança de quadro pode facilitar a resolução de um problema e/ou ajudar na compreensão de certo procedimento, inclusive quanto à prova de sua validade. Neste trabalho vamos apresentar quatro exemplos, em distintos contextos, e relacioná-los às caracterizações acerca do Jogo de quadros, não como necessidade para resolver um problema, mas para mostrar que em outro contexto cada um deles ganha outros olhares, seja para a melhor compreensão dos procedimentos envolvidos, seja para dimensionar o alcance dos conceitos.
\end{abstract}

Palavras-chave: Jogo de quadros. Mudança de quadro. Ensino de Matemática. Conhecimento. Saber.

\begin{abstract}
This work aims to reflect about issues related to teaching and learning of mathematical concepts. Often especially in geometry - when a problem is proposed is no need to change the context in which it is presented in order to solve it. As this passage from one context to another Régine Douady calls changing table (Game Tables). Sometimes making a change of frame can facilitate the resolution of a problem and / or help in understanding certain procedure, including as proof of its validity. In this work we present four examples, in different contexts, and relate them to characterizations about the game boards, not as a need to solve a problem, but to show that in another context each other looks win, whether for better understanding procedures involved, is to scale the range of concepts.
\end{abstract}

Keywords: Game of presentment. Change of presentment. Teaching of Mathematics. Knowledge. Knowing.

\section{Introdução}

O conceito de "quadro, mudança de quadro e jogo de quadros" foi utilizado na didática da matemática francesa pela primeira vez por Régine Douady (1986), na sua tese de doutoramento "Jogo de quadros e dialética ferramenta/objeto".

Muitas vezes - particularmente em problemas de geometria - quando um problema é proposto, para resolvê-lo é necessário mudar o contexto em que ele é apresentado de modo a poder resolvê-lo.

Essa passagem, de um contexto para outro, foi chamado por Régine Douady de mudança de quadro (Jogo de quadros). 
Por vezes, fazer uma mudança de quadro pode facilitar a resolução de um problema. Mas, também, pode ajudar na compreensão de certo procedimento que por vezes o professor apresenta e faz uso com seus alunos e, por estar em um contexto diferente daquele em que está sendo utilizado, ou então, por necessitar de outros conceitos matemáticos que justifiquem sua validade, o professor se vê impossibilitado de, naquele particular contexto, poder justificar para seus alunos sua adequação e validade.

Assim, neste caso, o procedimento é adequado para aquele contexto mas nele, o professor não dispõe de razões apropriadas/adequadas - dentro do contexto em que ele está sendo utilizado para apresentar a prova de sua validade, necessitando mudar de quadro (de contexto) para que possa fazê-lo.

Neste trabalho vamos apresentar quatro exemplos, em diferentes contextos - três deles da educação básica e um deles da álgebra linear, ensino superior - e relacioná-los às caracterizações presentes no Jogo de quadros.

Mas estes exemplos serão desenvolvidos, não como necessidade para resolver um problema em si, mas para mostrar que em outro contexto cada um deles ganha outros olhares seja para a melhor compreensão dos procedimentos que foram utilizados no desenvolvimento, seja para dimensionar o alcance dos conceitos apresentados de início.

É o caso do exemplo de álgebra linear, que iremos apresentar. Nele, conceitos associados às transformações lineares são consolidados mediante uma identificação com conceitos já consolidados da teoria das matrizes. Igualmente, conceitos identificados com as transformações lineares também podem ser definidos, de maneira similar, na teoria das matrizes.

Nestas situações, diz-se que fica estabelecido um isomorfismo (um operador linear bijetor) entre cada matriz de elementos reais e a correspondente transformação linear associada a ela.

\section{O que é um "quadro"?}

Douady (1992, p. 135) caracteriza a noção de quadro da seguinte maneira: "Um quadro é constituído de objetos de um campo da matemática, de relações entre esses objetos, de suas formulações eventualmente diferentes e das imagens mentais associadas a esses objetos e a essas relações". 
Temos como exemplos o quadro algébrico, o quadro geométrico, o quadro numérico, o quadro das funções, o quadro da estatística, o quadro vetorial, o quadro do cálculo numérico, etc...

Um quadro pode ser subdividido em outros quadros (Rogalski fala em sub-quadros) se o modelo teórico que o define (conjunto de axiomas da teoria) for diferente.

Assim por exemplo o quadro geométrico pode ser subdividido em quadro da geometria euclidiana, quadro da geometria hiperbólica, quadro da geometria esférica, quadro da geometria projetiva, quadro da geometria afim, quadro da geometria das transformações, quadro da perspectiva cônica, quadro da perspectiva cavaleira, quadro da geometria descritiva, quadro da geometria analítica, etc...

\section{O que é uma mudança de quadro?}

Segundo Douady (1992), uma mudança de quadro é uma passagem de um quadro para outro a fim de obter formulações diferentes de um problema. Esta mudança pode permitir uma nova entrada nas dificuldades encontradas e o funcionamento de ferramentas e técnicas não pertinentes na primeira formulação.

As mudanças de quadro podem ser espontâneas (iniciativa do aluno) ou provocadas (intervenção de outro aluno ou do professor)

Duval $^{1}$, no seu artigo "quadros e registros", diz que uma mudança de quadro é uma mudança de contexto, uma mudança de modelo teórico, uma mudança de direção do pensamento.

Utilizamos uma mudança de quadro sempre que num quadro o problema a ser solucionado ou a proposição a ser demonstrada apresenta uma grande dificuldade.

Assim pode-se mudar do quadro da geometria euclidiana para o quadro da geometria analítica ou vice-versa. Outras vezes pode se mudar do quadro das funções para o quadro geométrico. Dependendo do problema proposto, uma mudança de quadro pode ser necessária para obter a solução de uma questão ou pode apenas facilitar a resolução de um problema.

\section{O que é uma mudança de quadro?}

Um jogo de quadros consiste em trabalhar uma mesma questão de matemática em dois diferentes domínios da matemática. Assim, uma mudança de quadro é a passagem de um quadro para outro e a volta com o problema resolvido para o quadro inicial.

\footnotetext{
${ }^{1}$ Raymond Duval, filósofo e psicólogo francês, autor da Teoria dos Registros de Representações Semióticas.
} 
Os matemáticos, em geral, utilizam o jogo de quadros nas suas descobertas de resultados e correlações entre conceitos. O avanço de Régine Douady foi o de transportar para os alunos da Educação Básica ou Superior alguns elementos deste procedimento dos matemáticos, que pode ajudar na aprendizagem de novos conceitos e as relações entre diferentes conceitos da matemática.

Uma mudança de quadro se faz necessária quando existe uma grande dificuldade para resolver um problema ou demonstrar um resultado num determinado quadro no qual o conceito ou problema está sendo apresentado e, portanto, de modo a resolver esse impasse procura-se outro quadro onde as ferramentas matemáticas disponíveis facilitam a resolução do problema ou a demonstração.

Depois, uma vez resolvido o problema ou feita a demonstração, transporta-se a solução para o quadro inicial.

Douady (1992) sugere um jogo interativo entre quadros para fazer progredir os alunos na fase de busca de uma solução para um problema. Esse jogo de quadros consiste em mudanças de quadro provocadas por iniciativa do professor em certas situações as quais permitem fazer avançar o aluno na resolução do problema.

Trata-se do desenvolvimento de um procedimento no qual se transfere o problema de um quadro para outro, interpretam-se as correspondências entre os elementos dos dois quadros, resolve-se o problema e finalmente volta-se com a solução do problema para o quadro de partida.

Nesse procedimento para a busca da solução do problema, Douady distingue três fases: uma transferência de um quadro para o outro, onde nesse último é dada uma interpretação ao fenômeno a ser estudado; o estabelecimento de correspondências imperfeitas (seja por razões matemáticas ou por conhecimentos insuficientes por parte dos alunos) entre objetos e relações dos dois quadros; e finalmente uma melhoria entre essas correspondências permitindo o progresso do conhecimento de cada um dos quadros.

\section{Diferentes exemplos de Mudança de Quadros}

Exemplo 1:

O quadro da Aritmética de números naturais é constituído da construção do conjunto, de operações elementares e de resultados envolvendo esses elementos. 
Já o quadro da Teoria dos Conjuntos é constituído por relações de pertinência, de inclusão e operações entre conjuntos, entre outros. Se a axiomática que envolve os números naturais for considerada, o quadro inclui todos os resultados já matematicamente provados.

Para provar que o procedimento de determinação de todos os divisores de um número bastante utilizado nos livros didáticos da Educação Básica - "sempre funciona", mas se torna incipiente para resolver outras questões correlatas, precisamos lançar mão da ideia de mudança de quadros, segundo Douady (1992).

Queremos resolver o seguinte problema: Quantos divisores inteiros e positivos possui o número 12 ?

Uma parcela considerável de professores dos anos iniciais do Ensino Fundamental utiliza o seguinte processo para determinar quais são os divisores de 12 e como calcular o quantitativo desses divisores

Figura 1: Dispositivo prático para a determinação dos divisores de 12.

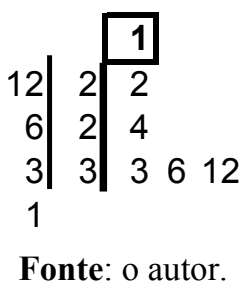

É um procedimento canônico bastante conhecido e utilizado pelos professores e, igualmente, está presente em muitos livros didáticos. Da maneira como o procedimento é apresentado e desenvolvido, ele funciona bastante bem para certo tipo de situações que envolvem a determinação de todos os divisores de um número e a totalidade deles por contagem direta, como é o caso do exemplo acima.

As questões que se colocam são: Porque esse procedimento funciona? Qual justificativa matemática está presente na sua utilização?

Depois de dividir o número pelos divisores primos em ordem monótona (iguais ou maiores do que) e, assim, "fatorar" o número dado segundo os números primos, o professor desenha um traço vertical e coloca o número 1 em destaque (Por que razão se faz assim? Porque é assim, costumam os professores referir-se a esse repetido hábito) e, em seguida, multiplicam-se os fatores primos do número dado pelos resultados anteriores. 
Por fim, o professor "informa" a seus alunos que, pelo fato de 12 ser escrito como $12=2^{2} .3^{1}$ então, para determinar o quantitativo de divisores de 12 basta tomar os expoentes dos fatores primos do número dado e, um a um, após somar uma unidade a cada um deles, multiplicá-los entre si, obtendo o total de divisores. Assim, para o caso do total de divisores de 12 tem-se: $(2+1) .(1+1)=3.2=6$ divisores. Mas, porque isso sempre funciona? Por que é feito assim?

De modo a provar que este procedimento funciona perfeitamente bem, vamos fazer uma Mudança de Quadros de maneira a provar que este procedimento é válido e encontra fundamentação teórica matemática para garantir que o resultado é verdadeiro.

Vejamos:

Um número a é divisor de 12 se e somente se a é da forma $\mathbf{a}=2^{m} \cdot 3^{n}, \mathrm{~m} \in\{0,1,2\} \mathrm{e}$ $\mathrm{n} \in\{0,1\}$ pois $12=2^{2} \cdot 3^{1}$.

Como 1 é divisor de qualquer número diferente de zero, então as escolhas $m=0$ e $n=0$ são as escolhas "triviais", ou seja, é a menor das escolhas para os expoentes de todos os fatores primos do número dado, quando combinados. Estas escolhas sempre serão possíveis de ser feitas, quando fatoramos o número que se deseja conhecer os divisores dele, e determinar a quantidade desses divisores.

Neste exemplo as escolhas recaem nos fatores primos 2 e 3 como $2^{m} \cdot 3^{n}=2^{0} \cdot 3^{0}=1$.

As outras opções de escolhas para os expoentes dos fatores primos 2 e 3 são as seguintes:

$$
\begin{aligned}
& \mathrm{m}=1 \text { e } \mathrm{n}=0 \text {, que determina o divisor } 2^{m} \cdot 3^{n}=2^{1} \cdot 3^{0}=2 ; \\
& \mathrm{m}=2 \text { e } \mathrm{n}=0 \text {, que determina o divisor } 2^{m} \cdot 3^{n}=2^{2} \cdot 3^{0}=4 ; \\
& \mathrm{m}=0 \text { e } \mathrm{n}=1 \text {, que determina o divisor } 2^{m} \cdot 3^{n}=2^{0} \cdot 3^{1}=3 ; \\
& \mathrm{m}=1 \text { e } \mathrm{n}=1 \text {, que determina o divisor } 2^{m} \cdot 3^{n}=2^{1} \cdot 3^{1}=6 ; \\
& \mathrm{m}=2 \text { e } \mathrm{n}=1 \text {, que determina o divisor } 2^{m} \cdot 3^{n}=2^{2} \cdot 3^{1}=12 \text {, e estas escolhas encerram }
\end{aligned}
$$
as escolhas possíveis.

A Tabela de dupla entrada a seguir ilustra a "combinação dos fatores primos 2 e 3 e seus possíveis expoentes": 
Figura 2: Tabela de dupla entrada com a combinação dos fatores primos de 12 e seus expoentes possíveis.

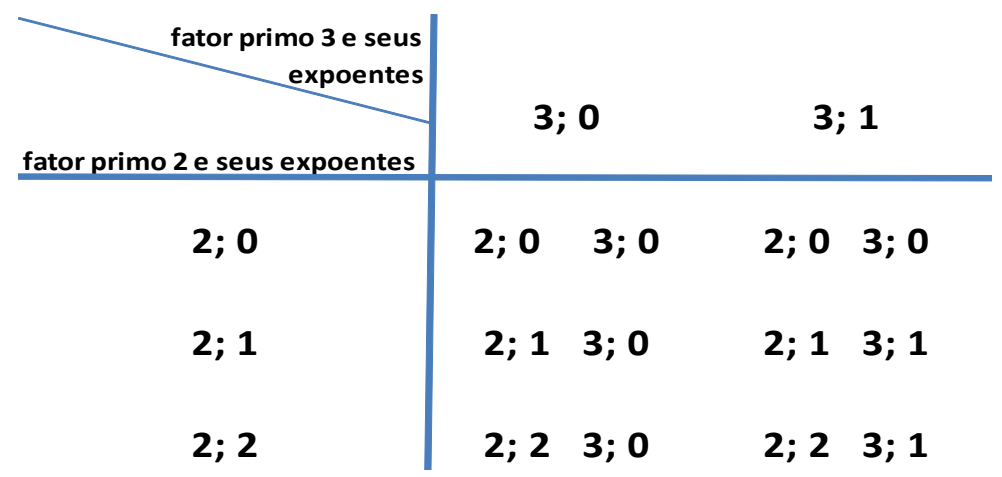

Fonte: o autor.

Portanto, o total de divisores do número 12 é 6 . O número de modos de escolher o expoente do fator primo 2 é 3, consoante sejam as escolhas 0,1 ou 2. O número de modos de escolher o expoente do fator primo 3 é 2, consoante sejam as escolhas 0 ou 1. Para cada escolha do expoente do fator primo 2 podemos combinar com uma escolha para o expoente do fator primo 3. Portanto, pelo Princípio Multiplicativo (ou Princípio da Multiplicação) da Análise Combinatória, tem-se que o número total de escolhas possíveis é $3.2=6$, correspondente ao total de divisores do número 12.

De modo similar ao método de colocar o número 1 em destaque, como visto no procedimento anterior, justifica-se essas escolhas de expoentes iguais a zero, sempre, como foi feito na observação anterior quando da escolha de $\mathrm{m}=0$ e de $\mathrm{n}=0$ (uma escolha imediata, natural).

Assim, se um número possui os fatores primos 2, 3, 5, 7, 11, etc, sempre haverá a escolha natural de todos os expoentes iguais a zero determinando, então, o valor $1=2^{0} \cdot 3^{0} \cdot 5^{0} \cdot 7^{0} \cdot 11^{0} \ldots \ldots$ Essa escolha é sempre possível, uma vez que o conjunto de opções dos expoentes dos fatores primos de um número é um conjunto de números naturais em que o zero é sempre um de seus elementos.

Por outro lado, fica claro que elevar qualquer fator primo do número dado à potência 1 e os demais fatores primos do número à potência zero determina todos os fatores primos do número dado que, obviamente, também são divisores imediatos, do número dado. No exemplo dado tem-se: $2^{1} \cdot 3^{0}=2$ e $2^{0} \cdot 3^{1}=3$ como os divisores primos diferentes de 1 .

Assim, para provar que o procedimento de fato "funciona", será preciso fazer uma Mudança de Quadros para o quadro da Análise Combinatória, onde é possível provar que o "procedimento" em tela nada mais é que um mecanismo para justificar as escolhas do zero como os expoentes dos fatores primos do número dado para determinar o divisor 1 , e os 
demais divisores do número são obtidos por combinação entre os possíveis expoentes dos fatores primos presentes no número.

Esse prova - por meio de conceitos da Análise Combinatória - é bastante enriquecedora no sentido de permitir responder a outras questões, dentro desse mesmo contexto de divisores de um número, como por exemplo:

- decidir se um determinado número é ou não divisor de outro sem que seja preciso determinar todos os divisores do número dado e comparar com o que foi dado para a pergunta, mas sim o de compreender de que modo o número é decomposto como produtos de seus fatores primos elevados aos possíveis expoentes - respondendo à pergunta tomando por base a possibilidade ou não da escolha de todos os expoentes do número que se deseja verificar se é divisor ou não para os respectivos fatores primos dos dois números.

Assim, por exemplo, responder se 8 é divisor de 12 se resume a fatorar o número 8 , o que dá $2^{3}$, e constatar que 3 não é uma possível escolha dentre as opções dos expoentes do fator primo 2 presente na fatoração do número 12, que são o 0 (zero), o 1 (um) ou o 2 (dois), pois $12=2^{2} \cdot 3^{1}$. Portanto, conclui-se que 8 não é divisor de 12 .

Suponha que, agora, se está diante da situação de determinar qual o menor número natural que tem 24 divisores. Como proceder?

O procedimento apresentado acima não é suficiente para resolver este problema. Nesse caso, deve-se decidir sobre as escolhas dos expoentes dos fatores primos 2, 3, 5 e 7, respectivamente os expoentes ${ }^{\beta},{ }_{\varphi},{ }^{\delta} \mathrm{e}_{\varrho}$, que satisfazem aos possíveis produtos dos expoentes somados com a unidade: $(\beta+1) \cdot(\varphi+1) \cdot(\delta+1) \cdot(e+1)=24$, tomando-se dois dos fatores primos, três fatores ou até os quatro fatores, e decidir sobre aqueles expoentes que determinam o menor número com 24 divisores.

Assim, possivelmente um aluno começaria a solução deste problema construindo a tabela abaixo (ou outra similar) para o uso de dois ou até quatro dos fatores primos $2,3,5$ ou 7, e obter o menor dos números que tem 24 divisores. 
Tabela 1: Determinação do menor número que possui 24 divisores.

\begin{tabular}{|l|l|l|l|l|l|l|}
\hline $\begin{array}{l}\text { 24 escrito } \\
\text { em 2, 3 ou } \\
\text { produtos. }\end{array}$ & $\begin{array}{l}\text { Expoente } \\
\text { fator } \\
\text { primo 2. }\end{array}$ & $\begin{array}{l}\text { Expoente } \\
\text { fator } \\
\text { primo 3. }\end{array}$ & $\begin{array}{l}\text { Expoente } \\
\text { fator } \\
\text { primo 5. }\end{array}$ & $\begin{array}{l}\text { Expoente } \\
\text { fator } \\
\text { primo 7. }\end{array}$ & $\begin{array}{l}\text { Número obtido, } \\
\text { contendo 24 } \\
\text { divisores. }\end{array}$ & Situação \\
\hline $24=3.2 .2 .2$ & 2 & 1 & 1 & 1 & $22.31 .51 .71=420$ & \\
\hline $24=2.3 .2 .2$ & 1 & 2 & 1 & 1 & $21.32 .51 .71=630$ & MAIOR \\
\hline $24=2.3 .4$ & 1 & 2 & 3 & 0 & $21.32 .53 .70=750$ & MAIOR \\
\hline $24=2.4 .3$ & 1 & 3 & 2 & 0 & $21.33 .52 .70=1350$ & MAIOR \\
\hline $24=3.2 .4$ & 2 & 1 & 3 & 0 & $22.31 .53 .70=1500$ & MAIOR \\
\hline $24=3.4 .2$ & 2 & 3 & 1 & 0 & $22.33 .51 .70=270$ & MENOR \\
\hline $24=4.2 .3$ & 3 & 1 & 2 & 0 & $23.31 .52 .70=600$ & MAIOR \\
\hline $24=4.3 .2$ & 3 & 2 & 1 & 0 & $23.32 .51 .70=360$ & MAIOR \\
\hline $24=3.8$ & 2 & 7 & 0 & 0 & $22.37 .50 .70=\ldots$ & MAIOR \\
\hline
\end{tabular}

Fonte: o autor.

Analisando os resultados obtidos na tabela acima, conclui-se que o menor número que tem 24 divisores é o número 270.

Vejamos agora como poderíamos proceder para determinar quantos são os números menores que 100 que têm 12 divisores. Como proceder?

Nesse caso, a exemplo do caso anterior, deve-se decidir sobre todas as escolhas dos expoentes dos fatores primos 2, 3, 5, etc que satisfazem aos possíveis produtos $(\beta+1) \cdot(\varphi+1) \cdot(\varphi+1)=12$ e que determinam os números com 12 divisores e menores que 100. 
Também aqui poderia ser feito uma mudança de quadros para a Análise Combinatória e a análise das possibilidades ser feita sobre as "folhas terminais" de uma árvore de possibilidades.

A tabela a seguir mostra as análises que são feitas.

Tabela 2: Determinação dos números menores que 100 que possuem 12 divisores.

\begin{tabular}{|c|c|c|c|c|c|}
\hline $\begin{array}{l}12 \text { escrito } \\
\text { em 2 ou 3 } \\
\text { produtos. }\end{array}$ & $\begin{array}{l}\text { Expoente } \\
\text { fator } \\
\text { primo 2. }\end{array}$ & $\begin{array}{l}\text { Expoente } \\
\text { fator } \\
\text { primo 3. }\end{array}$ & $\begin{array}{l}\text { Expoente } \\
\text { fator } \\
\text { primo 5. }\end{array}$ & $\begin{array}{l}\text { Número obtido, } \\
\text { com 12 divisores. }\end{array}$ & Situação \\
\hline $12=2.2 .3$ & 1 & 1 & 2 & $2^{1} \cdot 3^{1} \cdot 5^{2}=150$ & MAIOR \\
\hline $12=2.3 .2$ & 1 & 2 & 1 & $2^{1} \cdot 3^{2} \cdot 5^{1}=90$ & MENOR(*) \\
\hline $12=3.2 .2$ & 2 & 1 & 1 & $2^{2} \cdot 3^{1} \cdot 5^{1}=60$ & MENOR(*) \\
\hline $12=1.12$ & 0 & 11 & 0 & $2^{0} \cdot 3^{11} \cdot 5^{0}=\ldots \ldots$ & MAIOR \\
\hline $12=12.1$ & 11 & 0 & 0 & $2^{11} \cdot 3^{0} \cdot 5^{0}=\ldots \ldots$ & MAIOR \\
\hline $12=2.6$ & 1 & 5 & 0 & $2^{1} \cdot 3^{5} \cdot 5^{0}=486$ & MAIOR \\
\hline $12=6.2$ & 5 & 1 & 0 & $2^{5} \cdot 3^{1} \cdot 5^{0}=96$ & MENOR(*) \\
\hline $12=3.4$ & 2 & 3 & 0 & $2^{2} \cdot 3^{3} \cdot 5^{0}=108$ & MAIOR \\
\hline $12=4.3$ & 3 & 2 & 0 & $2^{3} \cdot 3^{2} \cdot 5^{0}=72$ & MENOR(*) \\
\hline
\end{tabular}

Fonte: o autor.

Analisando os resultados obtidos na tabela acima se conclui que os números 60, 72, 90 e 96 são todos os possíveis números menores que 100 que possuem 12 divisores.

Vejamos outra situação de Mudança de Quadros: Queremos determinar o número de modos de iluminar uma sala que possui 4 lâmpadas em perfeito estado de funcionamento.

De uma maneira simplificada podemos obter a solução após utilizar a enumeração de todas as possibilidades e contá-las. A seguir temos todas as possibilidades de iluminar a sala:

- iluminando a sala com uma única lâmpada acesa: L1, L2, L3 e L4, ou seja, quatro possibilidades;

- iluminando a sala com duas lâmpadas acesas: L1-L2, L1-L3, L1-L4, L2-L2, L2-L4 e L3-L4, ou seja, seis possibilidades; 
- iluminando a sala com três lâmpadas acesas: L1-L2-L3, L1-L2-L4, L1-L3-L4, e L2L3-L4, ou seja, quatro possibilidades;

- iluminando a sala com todas as quatro lâmpadas acesas: L1-L2-L3-L4, ou seja, uma única possibilidade.

Podemos obter a solução após construir árvores de possibilidades oriundas das ideias do Princípio Multiplicativo da Análise Combinatória e, assim, por enumeração de todas as possibilidades, determinar a solução.

A seguir temos a construção de quatro árvores de possibilidades mostrando as diferentes maneiras de iluminar a sala:

Figura 3: Árvore de possibilidades para a solução de 4.4.2.
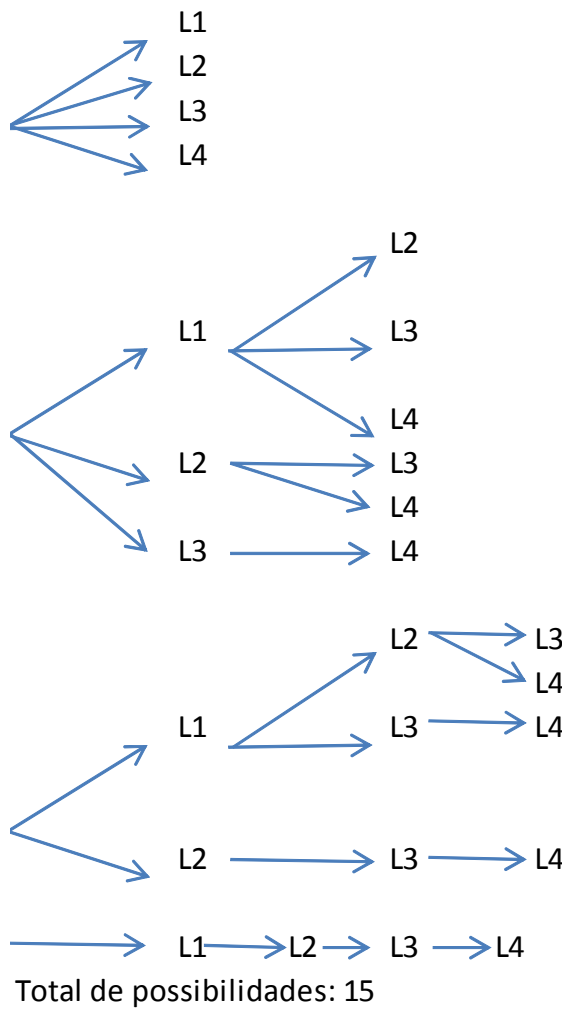

Fonte: o autor.

Assim, por enumeração das soluções do problema, se tem 15 diferentes maneiras de iluminar uma sala que dispõe de quatro lâmpadas.

Poderíamos fazer uma Mudança de Quadros para provar, de um outro modo, que o resultado é verdadeiro, agora se utilizando da Teoria dos Conjuntos. Vejamos:

Dado o conjunto $\mathrm{A}=\{\mathrm{L} 1, \mathrm{~L} 2, \mathrm{~L} 3, \mathrm{~L} 4\}$, o conjunto de partes desse conjunto é dado por: 


$$
\begin{aligned}
\wp(\mathrm{A})= & \{\varnothing,\{\mathrm{L} 1\},\{\mathrm{L} 2\},\{\mathrm{L} 3\},\{\mathrm{L} 4\},\{\mathrm{L} 1, \mathrm{~L} 2\},\{\mathrm{L} 1, \mathrm{~L} 3\},\{\mathrm{L} 1, \mathrm{~L} 4\},\{\mathrm{L} 2, \mathrm{~L} 3\},\{\mathrm{L} 2, \mathrm{~L} 4\}, \\
& \{\mathrm{L} 3, \mathrm{~L} 4\},\{\mathrm{L} 1, \mathrm{~L} 2, \mathrm{~L} 3\},\{\mathrm{L} 1, \mathrm{~L} 2, \mathrm{~L} 4\},\{\mathrm{L} 1, \mathrm{~L} 3, \mathrm{~L} 4\},\{\mathrm{L} 2, \mathrm{~L} 3, \mathrm{~L} 4\},\{\mathrm{L} 1, \mathrm{~L} 2, \mathrm{~L} 3, \mathrm{~L} 4\}\} .
\end{aligned}
$$

A quantidade de elementos do conjunto de partes é dada por

$\# \wp(\mathrm{A})=2^{4}=16$. Como devemos ter ao menos uma lâmpada acesa de modo que a sala fique iluminada, o conjunto $\varnothing$ não deve ser considerado, em função de representar o fato de que todas as lâmpadas estão desligadas. Assim, tem-se $2^{4}-1=16-1=15$ possibilidades de iluminar a sala.

Poderíamos fazer uma nova Mudança de Quadros para provar, de um outro modo, que o resultado é verdadeiro, agora se utilizando as propriedades do Triângulo de Pascal.

Sabe-se que o somatório dos elementos da n-ésima linha do Triângulo de Pascal é dado por 2 ${ }^{n}$. No caso particular deste exemplo, considerando a $4^{\text {a }}$ linha do triângulo de Pascal, para o fato de dispor de 4 lâmpadas na sala, esse somatório é dado por $2^{4}=16=C^{4,0}+C^{4,1}+C^{4,2}+$ $+\mathrm{C}^{4,3}+\mathrm{C}^{4,4}$

Cada uma das parcelas pode ser entendida como $\mathrm{C}^{4, p}$, ou seja, o número de modos de acender $\mathrm{p}$ lâmpadas entre 4 lâmpadas disponíveis, onde $\mathrm{p} \in\{0,1,2,3,4\}$. Mas, o coeficiente binomial $C^{4,0}$ indica a opção de, entre as 4 lâmpadas disponíveis, não ter aceso nenhuma delas o que, evidentemente, não atende à solução do problema.

Assim, esta opção não deve ser considerada e deve ser retirada. Portanto, o total de possibilidades é dado por $16-1=15$.

Poderíamos fazer uma nova Mudança de Quadros para provar, de um outro modo, que o resultado é verdadeiro, novamente utilizando conceitos oriundos do Princípio Multiplicativo.

Há duas possibilidades para a lâmpada L1: estar acesa ou apagada. O mesmo se aplica para as demais lâmpadas da sala. Assim, o total de possibilidades é dado por 2.2.2.2 = 16 possibilidades.

Mas, dentre essas 16 possibilidades, incluímos a possibilidade de que as quatro lâmpadas estejam todas apagadas. Logo, desse total de 16 possibilidades é preciso retirar essa possibilidade (todas as lâmpadas apagadas) ficando, então, com 15 distintos modos de ter a sala acesa quando se tem 4 lâmpadas. 
Esse resultado pode ser generalizado para um quantitativo de $\mathrm{n}$ lâmpadas em uma sala e queremos calcular o total de diferentes modos de iluminar essa sala, o que dá um total de $2^{\text {n }}$ 1 diferentes modos de ter a sala iluminada.

\section{Exemplo 3:}

Vamos avaliar uma mudança de quadro para o quadro da álgebra linear, apresentado a seguir.

Considere o sistema linear

$$
\left[\begin{array}{c}
a_{11} x_{1}+\ldots \ldots \ldots \ldots+a_{1 n} x_{n}=b_{1} \\
\cdot \\
a_{m 1} x_{1}+\ldots \ldots \ldots \ldots+a_{m n} x_{n}=b_{m}
\end{array}\right.
$$

A matriz ampliada desse sistema é formada pela matriz dos coeficientes das variáveis e a matriz dos termos independentes:

$$
\left[\begin{array}{cccc}
a_{11} & \cdots & a_{1 n} & b_{1} \\
\vdots & \ddots & \vdots & \\
a_{m 1} & \cdots & a_{m n} & b_{m}
\end{array}\right]
$$

Assim, o sistema linear pode ser representado pelo produto matricial A.X $=\mathrm{B}$, onde $A_{m x n}$ é a matriz $\left[\begin{array}{ccc}a_{11} & \cdots & a_{1 n} \\ \vdots & \ddots & \vdots \\ a_{m 1} & \cdots & a_{m n}\end{array}\right], B_{m x 1}$ é a matriz $\left[\begin{array}{c}b_{1} \\ \\ b_{m}\end{array}\right]$ e $X_{n x 1}$ é a matriz $\left[\begin{array}{c}x_{1} \\ x_{n}\end{array}\right]$.

O sistema linear acima pode ser encarado como a equação vetorial

$$
X_{1}\left(a_{11}, a_{21}, \ldots \ldots ., a_{m 1}\right)+\ldots \ldots \ldots \ldots . .+X_{n}\left(a_{1 n}, a_{2 n}, \ldots \ldots \ldots . ., a_{m n}\right)=\left(b_{1}, \ldots \ldots . ., b_{m}\right) .
$$

A matriz $A_{m x n}$ é encarada como uma aplicação linear $T_{A}: R^{n} \rightarrow R^{m}$ em relação às bases canônicas $\beta=\left\{v_{1}, \ldots \ldots, v_{n}\right\}$ de $R^{n}$ e $\beta^{\prime}=\left\{w_{1}, \ldots \ldots, w_{m}\right\}$ de $R^{m}$.

Diz-se também que podemos associar a matriz $A_{m x n}$ como:

$$
\begin{aligned}
& T_{A}: R^{n} \rightarrow R^{m} \\
& v \rightarrow T_{A}(v) .
\end{aligned}
$$

Seja $\quad \mathrm{X}=[v]_{\beta}=\left[\begin{array}{l}x_{1} \\ \\ x_{n}\end{array}\right]$ e $T_{A}(v)=\mathrm{A} \cdot \mathrm{X}=\left[\begin{array}{ccc}a_{11} & \cdots & a_{1 n} \\ \vdots & \ddots & \vdots \\ a_{m 1} & \cdots & a_{m n}\end{array}\right] \cdot\left[\begin{array}{l}x_{1} \\ x_{n}\end{array}\right]=\left[\begin{array}{l}y_{1} \\ y_{n}\end{array}\right]$.

Então, $T_{A}(v)=\mathrm{y}_{1} \mathrm{w}_{1}+\ldots \ldots \ldots+\mathrm{y}_{\mathrm{m}} \mathrm{w}_{\mathrm{m}}$, onde $\mathrm{y}_{\mathrm{i}}=A_{i} . X$ e $A_{i}$ é a i-ésima linha de A.

Em geral, 


$$
\begin{gathered}
T_{A}(u+v)=\mathrm{A} \cdot(u+v)=\mathrm{A} u+\mathrm{A} v=T_{A}(u)+T_{A}(v) \\
T_{A}(\alpha u)=\mathrm{A} .(\alpha u)=\alpha(\mathrm{A} u)=\alpha T_{A}(u) .
\end{gathered}
$$

Logo, $T_{A}: R^{n} \rightarrow R^{m}$

$v \rightarrow T_{A}(v)$ é transformação linear.

Assim, dada uma matriz $A_{m x n}$, ela produz uma transformação linear

$$
T_{A}: R^{n} \rightarrow R^{m}
$$

$v \rightarrow T_{A}(v)$ em relação às bases canônicas $\beta=\left\{v_{1}, \ldots \ldots, v_{n}\right\}$ de $R^{n}$ e $\beta^{\prime}=\left\{w_{1}, \ldots \ldots, w_{m}\right\}$ de $R^{m}$.

Neste caso, muda-se o quadro associado aos conceitos presentes na teoria das matrizes e dos sistemas lineares para o quadro da álgebra linear, particularmente, o estudo das transformações lineares.

Por outro lado, dada uma transformação linear T: $R^{n} \rightarrow R^{m}$ ela pode ser representada por uma matriz $\quad[T]_{\beta^{\prime}}^{\beta}$ relativamente às bases $\beta=\left\{v_{1}, \ldots \ldots, v_{n}\right\}$ e $\beta^{\prime}=\left\{w_{1}, \ldots \ldots, w_{m}\right\}$, respectivaente, de $R^{n}$ e de $R^{m}$, tal que

$$
\left\{\begin{array}{c}
T\left(v_{1}\right)=u_{1}=\sum_{i=1}^{m} a_{i 1} \cdot w_{i} \\
\vdots \\
T\left(v_{n}\right)=u_{n}=\sum_{i=1}^{m} a_{i m} \cdot w_{i}
\end{array}\right\}
$$

Daí: $[T]_{\beta \prime}^{\beta}=\left[\begin{array}{ccc}a_{11} & \cdots & a_{1 m} \\ \vdots & \ddots & \vdots \\ a_{n 1} & \cdots & a_{n m}\end{array}\right]$ é a matriz de $\mathrm{T}$ em relação às bases canônicas $\beta=$ $\left\{v_{1}, \ldots \ldots, v_{n}\right\}$ de $R^{n}$ e $\beta^{\prime}=\left\{w_{1}, \ldots \ldots, w_{m}\right\}$ de $R^{m}$.

Além do mais, a matriz $[T]_{\beta}^{\beta}$, é a transposta da matriz dos coeficientes do sistema linear apresentado acima, ou seja, $A_{m x n}=\left[\begin{array}{ccc}a_{11} & \cdots & a_{1 n} \\ \vdots & \ddots & \vdots \\ a_{m 1} & \cdots & a_{m n}\end{array}\right]$. Portanto, T passa a ser a aplicação linear associada à matriz $\mathrm{A}$ e bases $\beta=\left\{v_{1}, \ldots \ldots, v_{n}\right\}$ e $\beta^{\prime}=\left\{w_{1}, \ldots \ldots, w_{m}\right\}$, isto é, $\mathrm{T}=T_{A}$.

Portanto, propriedades que a matriz $A_{m x n}$ possui podem ser associadas às propriedades da transformação linear associada a ela, como por exemplo:

- $\operatorname{dim} \operatorname{Im}(\mathrm{T})=\mathrm{p}=$ posto da matriz $[T]_{\beta}^{\beta}=$ número de linhas não nulas da matriz $\mathrm{B}_{\mathrm{mxn}}, \mathrm{a}$ matriz-linha reduzida à forma escada linha equivalente a $A_{m x n}$. 
$-\operatorname{dim} \operatorname{Ker}(\mathrm{T})=\operatorname{dim} \operatorname{Nuc}(\mathrm{T})=$ nulidade da matriz $[T]_{\beta \prime}^{\beta}=\mathrm{n}-\mathrm{p}=$ diferença entre o número de colunas da matriz $A_{m x n}$ e o posto da matriz $[T]_{\beta \prime}^{\beta}$.

- a transformação linear T: $V \rightarrow W$ é inversível se e somente se $\operatorname{det}[T]_{\beta \prime}^{\beta} \neq 0$.

- se a transformação linear T: $V \rightarrow W$ é inversível então $T^{-1}:: \mathrm{W} \rightarrow \mathrm{V}$ é operador linear e $\left[T^{-1}\right]_{\beta}^{\beta^{\prime}}=\left([T]_{\beta \prime}^{\beta}\right)^{-1}$.

Essa mudança de quadro da álgebra linear permite que todo o estudo dos operadores lineares, aí associados os conceitos de autovalores e autovetores, os operadores auto-adjuntos e ortogonais, as formas lineares, bilineares e quadráticas e a classificação de cônicas e quádricas podem ser feitas com as ferramentas associadas à teoria das matrizes e dos determinantes, configurando-se em valioso instrumento para a exploração de conceitos presentes nas cônicas, ou seções cônicas e nas quádricas, por exemplo, estudadas pelos gregos e desenvolvidas a partir de suas propriedades geométricas, presentes nos trabalhos de Apollonius (260-170 A.C.).

\section{Exemplo 4:}

Vejamos outra situação de Mudança de Quadros: Queremos encontrar um modo de determinar o número total de diagonais de um polígono regular de $\mathrm{n}$ lados, $\mathrm{n} \geq 3$.

Poderíamos fazer assim: Escolhido um vértice qualquer $\mathrm{v}_{1}$, os segmentos de reta que unem os vértices adjacentes a esse vértice $\left(\mathrm{v}_{2} \mathrm{e} \mathrm{v}_{n}\right)$ são lados do polígono e, assim, não podem ser contados como diagonais.

Por outro lado, sobram (n-3) vértices do polígono cujos segmentos de reta que os unem ao vértice $\mathrm{v}_{1}$ são diagonais do polígono. Portanto, a partir desse vértice podemos traçar (n-3) diagonais. O mesmo ocorre para quaisquer um dos outros (n-1) vértices do polígono. Assim, traçamos (n-3).n diagonais.

Mas, todas essas diagonais foram duplamente contadas quando se conta as diagonais a partir de cada uma das extremidades dessas diagonais, o que evidencia uma contagem em dobro.

De modo a obtermos a contagem real, dividimos esse total por 2. Assim, há

$\frac{(n-3) \cdot n}{2}$ diagonais em um polígono regular de $\mathrm{n}$ vértices (correspondentemente $\mathrm{n}$ lados), $\mathrm{n}$ $\geq 3$. 
Poderíamos fazer uma Mudança de Quadros para provar, de outro modo, que o resultado acima é verdadeiro. Vejamos:

Se um polígono regular tem $\mathrm{n}$ lados, ao escolher dois quaisquer de seus vértices e uni-los por meio de um segmento de reta podemos obter uma diagonal ou um lado desse polígono.

Por outro lado, o número total de modos de fazer a escolha de 2(dois) quaisquer vértices é determinado por $\mathrm{C}_{n, 2}$ (número de modos de escolher dois objetos dentre $\mathbf{n}$ objetos distintos dados (combinações simples dois a dois)).

Assim, esse total $\mathrm{C}_{n, 2}$ de escolhas define, unicamente, o total de diagonais acrescido do número de lados do polígono de $\mathbf{n}$ vértices, ou seja: $\mathrm{C}_{n, 2}=$ número de diagonais $(\mathrm{d})+$ número de lados (n).

Portanto, $\mathrm{d}=\mathrm{C}_{n, 2}-\mathbf{n}=\frac{n \cdot(n-1)}{2}-n=\frac{n \cdot(n-3)}{2} ., \mathrm{n} \geq 3$.

Poderíamos fazer uma nova Mudança de Quadros para provar, de um outro modo - por Indução no número de vértices (respectivamente lados) - que o resultado é verdadeiro. Vejamos:

O polígono regular com o menor número de vértices é o triângulo, que possui 3 vértices e, portanto, não possui nenhuma diagonal.

Assim vale a primeira propriedade de indução P1, pois, para $\mathrm{n}=3$ tem-se que $\mathrm{d}_{3}=\frac{3 \cdot(3-3)}{2}=0$.

Considere agora (por hipótese de indução) que o resultado é válido para $\mathrm{n}=\mathrm{k}$. Assim, $\mathrm{d}_{k}=\frac{k \cdot(k-3)}{2}$. Vamos acrescentar um vértice $\mathrm{v}_{k+1}$ ao polígono anterior entre os vértices $\mathrm{v}_{j} \mathrm{e} \mathrm{v}_{j+1}, 1 \leq \mathrm{j} \prec \mathrm{n}$.

Desse novo vértice traçam-se $\mathrm{k}$ - 2 novas diagonais (exceto para os vértices vizinhos ao vértice $\mathrm{v}_{k+1}$, que são os vértices $\mathrm{v}_{j} \mathrm{e} \mathrm{v}_{j+1}$ ) e mais uma diagonal (que conecta os vértices $\mathrm{v}_{j} \mathrm{e}$ $\mathrm{v}_{j+1}$. Note que no polígono anterior, ao conectar os vértices $\mathrm{v}_{j} \mathrm{e} \mathrm{v}_{j+1}$ tínhamos um lado. Assim, no novo polígono tem-se $(\mathrm{k}-2)+1=\mathrm{k}-1$ diagonais a mais do que no polígono anterior. 
Portanto: $\mathrm{d}_{k+1}=(\mathrm{k}-1)+\frac{k \cdot(k-3)}{2}=$

$$
=\frac{2 k-2+k^{2}-3 k}{2}=\frac{k^{2}-k-2}{2}=\frac{(k-2) \cdot(k+1)}{2}=\frac{(k+1) \cdot[(k+1)-3]}{2} .
$$

Portanto, o resultado fica provado, por indução.

Vejamos uma outra situação de Mudança de Quadros: Queremos estender a ideia anterior para um poliedro regular de $\mathrm{n}$ vértices de modo a determinar o total de diagonais internas de poliedros regulares.

A escolha de dois quaisquer de seus vértices (cuja totalidade é dada por $\mathrm{C}_{n, 2}$ ) e uni-los por meio de um segmento de reta pode acarretar uma das três situações seguintes: ou obtém-se uma diagonal de face, ou uma diagonal interna do poliedro ou então uma aresta do poliedro.

Assim, $\mathrm{C}_{n, 2}=$ número de diagonais de face $(\mathbf{D F})+$ número de diagonais internas $(\mathbf{D I})+$ número de arestas (A).

Logo: número de diagonais internas $(\mathbf{D I})=\mathrm{C}_{n, 2}$ - número de diagonais de face $(\mathbf{D F})$ - número de $\operatorname{arestas}(\mathbf{A})$, ou seja: $\mathbf{D I}=\mathrm{C}_{n, 2}-\mathbf{D F}-\mathbf{A}$.

Vejamos o seguinte exemplo: Quantas são as diagonais internas de um octaedro?

As oito faces do octaedro são formadas por triângulos equiláteros que, obviamente, não têm diagonais de face. Além disso, o octaedro possui 6 vértices e 12 arestas (as quatro arestas da parte de cima, as quatro da parte de baixo e as quatro ao redor dos quatro vértices na parte central).

Assim: DI $=\mathrm{C}_{6,2}-0-12=15-0-12=3$, ou seja, o octaedro possui três diagonais internas, que são as seguintes: a diagonal que une os dois vértices isolados e as duas diagonais que unem, dois a dois, os vértices que estão na parte central.

De modo similar, prova-se que um tetraedro não possui diagonais internas, pois: $\mathbf{D I}=\mathrm{C}_{4,2}-0$ (as faces são triangulares) - 6 (as três arestas que ligam os vértices da base ao vértice de cima mais as três arestas que ligam os três vértices da base) $=6-0-6=0$.

O quadro abaixo mostra o total de diagonais internas para cada um dos Poliedros de Platão: 
Quadro 1: Determinação do total de diagonais internas dos Poliedros de Platão

\begin{tabular}{|c|c|c|c|c|c|c|c|c|}
\hline Poliedro & $\begin{array}{l}\text { número de } \\
\text { vértices }(\mathrm{V})\end{array}$ & $\begin{array}{l}\text { número } \\
\text { de faces }\end{array}$ & $\begin{array}{l}\text { polígono } \\
\text { das faces }\end{array}$ & $\begin{array}{c}\text { número de diago- } \\
\text { nais por face }\end{array}$ & $\begin{array}{c}\text { total de diagonais } \\
\text { de face (DF) }\end{array}$ & $\begin{array}{c}\text { número } \\
\text { de } \operatorname{arestas}(\mathrm{A})\end{array}$ & $C_{v, 2}$ & $\begin{array}{c}\mathrm{D}=\mathrm{C} \mathrm{V}, 2-\mathrm{A}-\mathrm{DF} \\
\text { diagonais internas }\end{array}$ \\
\hline Tetraedro & 4 & 4 & triângulo & 0 & $4 \times 0=0$ & $(4 \times 3) / 2=6$ & 6 & 0 \\
\hline Octaedro & 6 & 8 & triângulo & 0 & $8 \times 0=0$ & $(8 \times 3) / 2=12$ & 15 & 3 \\
\hline Cubo & 8 & 6 & quadrado & 2 & $6 \times 2=12$ & $(6 \times 4) / 2=12$ & 28 & 4 \\
\hline Dodecaedro & 20 & 12 & pentágond & 5 & $12 \times 5=60$ & $(12 \times 5) / 2=30$ & 190 & 100 \\
\hline Icosaedro & 12 & 20 & triângulo & 0 & $20 \times 0=0$ & $(20 \times 3) / 2=30$ & 435 & 405 \\
\hline
\end{tabular}

Fonte: $\mathrm{O}$ autor.

Vejamos outra situação: Queremos determinar uma maneira de contabilizar o número total de diagonais internas de um prisma regular reto que tem em suas bases um polígono regular de $\mathrm{n}$ lados.

Poderíamos fazer assim: Esse prisma possui n retângulos, congruentes entre si, que formarão as $\mathbf{n}$ faces laterais do prisma. Como cada retângulo possui duas diagonais tem-se, então, um subtotal de $2 n$ diagonais de face, contabilizadas no total de faces laterais do prisma.

As duas faces (superior e inferior) são formadas por um polígono regular de $\mathrm{n}$ lados, $\mathrm{n} \geq 3 \mathrm{e}$ portanto, cada uma das faces tem um total de $\mathrm{d}=\frac{(n-3) \cdot n}{2}$ diagonais.

Assim, há um subtotal de (n-3). n diagonais de face, contabilizadas nas duas bases.

Portanto, tem-se o total de $2 n+(n-3)$. $n$ diagonais de face em um prisma regular reto que tem em suas bases um polígono regular de $\mathrm{n}$ lados.

Agora vamos determinar o total de diagonais internas desse prisma regular reto.

Inicialmente vamos numerar os $\mathbf{n}$ vértices da face superior e os $\mathbf{n}$ vértices da face inferior, obedecendo à mesma ordem dentre os índices desses vértices, isto é, para um dado vértice $\mathrm{v}_{j}$ da face superior, a aresta que o conecta com a face inferior encontra, exatamente, logo abaixo, $\mathrm{o}$ vértice $\mathrm{w}_{j}$.

Assim, escolhido um vértice qualquer $\mathrm{v}_{j}$ da face superior, os segmentos de reta que unem os vértices da face inferior "próximos deste vértice" - que são os vértices adjacentes $\mathrm{w}_{j-1}, \mathrm{w}_{j} \mathrm{e}$ $\mathrm{w}_{j+1}$ - quando conectados ao vértice $\mathrm{v}_{j}$ definem as seguintes situações: uma aresta $\left(\mathrm{v}_{j}, \mathrm{w}_{j}\right)$ ou então as diagonais de face $\left(\mathrm{v}_{j}, \mathrm{w}_{j-1}\right) \mathrm{e}\left(\mathrm{v}_{j}, \mathrm{w}_{j+1}\right)$ (lembre que essas diagonais já foram incluídas na contagem anterior). 
Portanto, escolhido um vértice $\mathrm{v}_{j}$ na face superior, há $(\mathrm{n}-3)$ vértices na face inferior que quando conectados ao vértice $\mathrm{v}_{j}$ da face superior definem diagonais internas. Portanto, contabilizando o total de diagonais internas para todos os vértices da face superior há um total de n.(n-3) diagonais internas.

Se repetíssemos o procedimento para os vértices localizados na face inferior, estaríamos contando, novamente, essas mesmas diagonais internas, já contadas anteriormente.

Então, há um total de $2 n+(n-3)$. $n$ diagonais de face e $n .(n-3)$ diagonais internas, totalizando $2[n+n .(n-3)]=2\left[n^{2}-2 n\right]=2 n .[n-2]$ diagonais de um prisma cujas bases são formadas por polígonos regulares de $\mathrm{n}$ vértices.

Mais uma vez poderíamos fazer uma Mudança de Quadros para provar - de uma outra maneira - que o resultado acima é verdadeiro. Vejamos:

Se o prisma regular tem $2 n$ vértices, ao escolher dois quaisquer de seus vértices e uni-los por meio de um segmento de reta, podemos obter uma das três situações: uma diagonal de face, uma aresta ou uma diagonal interna desse poliedro.

Por outro lado, o número de modos de fazer a escolha de 2(dois) quaisquer vértices, como já visto, é determinado por $\mathrm{C}_{2 n, 2}$ (número de modos de escolher dois objetos dentre os $2 \mathrm{n}$ objetos distintos dados (combinações simples)).

Assim, esse total de escolhas $\left(\mathrm{C}_{2 n, 2}\right)$ define, unicamente, o total de diagonais de face mais o total de arestas mais o total de diagonais internas desse poliedro. Ou seja: $\mathrm{C}_{2 n, 2}=$ número de diagonais de face $(\mathrm{DF})+$ número de $\operatorname{arestas}(\mathrm{A})+$ número de diagonais internas $(\mathrm{DI})$.

Portanto, $\mathrm{DI}+\mathrm{DF}=\mathrm{C}_{2 n, 2}-\mathrm{A}(*)$.

O número total de arestas é calculado como: $n$ arestas que conectam os vértices da face inferior aos vértices da face superior mais as $2 n$ arestas (que são os lados dos dois polígonos regulares das faces superior e inferior) dos dois polígonos regulares que constituem o prisma, totalizando $3 n$ arestas.

Assim, de (*) tem-se:

$$
\mathrm{DI}+\mathrm{DF}=\frac{2 n(2 n-1)}{2}-3 n=\frac{4 n^{2}-2 n-6 n}{2}=\frac{4 n^{2}-8 n}{2}=\frac{2 n^{2}-4 n}{1}=2 n(n-2) .
$$


Se quiséssemos calcular o total de diagonais internas (DI), poderíamos, utilizando a igualdade $(* *)$, proceder como a seguir:

Primeiramente vamos determinar o total de diagonais de face: As faces são de dois tipos: retângulos (as faces laterais) e polígonos regulares de $\mathrm{n}$ lados (a face superior e a face inferior do prisma).

Nos retângulos, há um total de: $n \cdot\left[C_{4,2}-4\right]=n \cdot[6-4]=2 n$ diagonais de face.

Nos polígonos regulares (faces superior e inferior) há um total de:

$$
\text { 2. } \frac{(n-3) \cdot n}{2}=n \cdot(n-3)=n^{2}-3 n \text { diagonais de face. }
$$

Logo, o total de diagonais de face é dado por $2 n+n^{2}-3 n=n^{2}-n$.

Portanto, de $(* *)$ tem-se que: DI $=2 n .(n-2)-\left(n^{2}-n\right)=2 n^{2}-4 n-n^{2}+n=n^{2}-3 n=n .(n-3)$, como provado anteriormente.

\section{Considerações finais}

Com frequência uma mudança de quadros vem acompanhada de mudança de registro. Uma mudança de registro coincide, em muitos casos, com uma mudança de quadro.

Nas situações-problema que foram apresentadas identificam-se diferentes registros de representação semiótica ${ }^{2}$, alguns deles dentro do mesmo quadro da Análise Combinatória e os outros no quadro da Teoria dos Conjuntos e no da Álgebra Linear.

A mudança de solução de qualquer uma dessas situações para o caso inicial ou vice-versa, representa uma Mudança de Quadros.

Quando se obtém, numa mesma situação, uma Mudança de Quadros e, a seguir, por uma nova Mudança de Quadros retorna-se ao quadro anterior, diz-se que fizemos uso de um Jogo de Quadros.

Para descrever uma mudança de quadro é preciso se centrar essencialmente sobre uma situação-problema, enquanto que para descrever uma mudança de registro é preciso se centrar essencialmente sobre os objetos representados.

\footnotetext{
2 Raymond Duval, filósofo e psicólogo francês, fornecem-nos um referencial estruturado de análise do funcionamento cognitivo da compreensão em matemática através da Teoria dos Registros de Representações Semióticas. Os objetos matemáticos não são diretamente acessíveis à percepção, o seu acesso passa, necessariamente, por representações semióticas (formas sob a qual a informação é descrita).
} 
Assim, por exemplo, segundo Raymond Duval, a compreensão conceitual sobre o objeto matemático função só é alcançada quando se coordena os registros algébricos, tabular e gráfico.

Esta é a condição para que o objeto não seja confundido com a sua representação e, por essa razão, considera-se que a passagem entre registros não se constitui em opção pedagógica do professor que permita enriquecer a resolução de uma situação-problema, mas que ela deva ser considerada obrigatória.

Entendemos que se trata de uma aprendizagem que não pode ser deixada de lado por conta de um aluno, mas que, necessária e obrigatoriamente, deva ser um objeto de ensino.

Por essas razões não há um acesso direto ao objeto matemático separado das suas representações, quando se deseja um conhecimento abrangente e aprofundado do objeto matemático em questão.

Com as considerações que foram apresentadas neste artigo, consideramos que a mudança de quadros deve ser incentivada pelos professores junto de seus alunos quando da proposição de situações-problema pertinentes.

\section{Referências}

DOUADY, Régine. Jeux de cadres et dialectique outil-objet dans l'enseignement des mathématiques. Tese (Doutorado). 1986.

DOUADY, Régine. Repères - IREM. Nº 6 - Janvier 1992.

DUVAL, Raymond. Basic Issues for Research in Mathematics Education. In: conference of the international group for the psycology of mathematics education, 24, 2000, Hiroshima. Proceedings of the $24^{\text {th }}$ PME. Hiroshima: Departament of Mathematics Education Hiroshima University, 2000, p. 55-69.

DUVAL, Raymond. Registros de representações semióticas e funcionamento cognitivo da compreensão em Matemática. In: MACHADO, S.D.A. Aprendizagem em Matemática: Registros de representação semiótica. Campinas: Papirus, 2003. P. 11-33.

DUVAL, Raymond. A cognitive analysys of problems of comprehension in a learning of mathematics. Educational Studies in Mathematics, Springer, 61: 103-131, 2006.

TEIXEIRA, Paulo Jorge Magalhães. Um estudo sobre os conhecimentos necessários ao professor de matemática para a exploração de problemas de contagem no Ensino Fundamental. Tese (Doutorado em Educação Matemática) - Universidade Bandeirante de São Paulo - UNIBAN, São Paulo, 2012. 